\title{
Integrated System for Objective Assessment of Global and Regional Lung Structure
}

\author{
J.M. Reinhardt ${ }^{1}$, J. Guo ${ }^{2}$, L. Zhang ${ }^{1}$, D. Bilgen ${ }^{1}$, S. Hu ${ }^{1,5}$, R. Uppaluri ${ }^{3,5}$, \\ R.M. Long ${ }^{3}$, O.I. Saba ${ }^{1}$, G. McLennan ${ }^{4}$, M. Sonka ${ }^{3}$, and E.A. Hoffman ${ }^{2,1}$ \\ 1 Department of Biomedical Engineering \\ 2 Department of Radiology \\ 3 Department of Electrical and Computer Engineering \\ 4 Department of Internal Medicine \\ University of Iowa, Iowa City, IA 52242 \\ ${ }^{5}$ G.E. Medical Systems, Milwaukee, WI 53201
}

\begin{abstract}
Sub-second multi-slice CT scanners can now provide detailed pulmonary structural and functional information. We describe an integrated software system to facilitate quantitative analysis of pulmonary anatomy and physiology. This system includes tools for lung, airway, lung lobe segmentation, parenchymal tissue characterization, as well as regional pulmonary ventilation and perfusion.
\end{abstract}

We have developed an integrated system for objective assessment of the lung from volumetric CT images. The system includes analysis tools to study lung structure via image segmentation and analysis applications that analyze the structure and function depicted in the images. The overall system architecture is shown in Figure 1. The inputs to the system are volumetric X-ray CT data (three or four dimensional) of the thorax. Processing is started by lung segmentation, followed by airway tree and lung lobe segmentation. Following segmentation, measurements are made for regions of interest, which can be automatically defined using anatomic landmarks. Lung and lobar volumes are computed. The airway tree topology is described by the branchpoints; additional measurements for the tree branches include cross-sectional area along branch; branch diameters and wall thicknesses as a function of branch generation; wall thickness uniformity along a branch and across branches of the same generation; volume of air and wall tissue along individual airway branches expressed per branch, per lobe, and per lung. The segmentation processing provides a structural decomposition that can be used for regional reporting of image-based measurements of lung anatomy or physiology (ventilation, perfusion, airway reactivity, etc.). A database is used to store intermediate and final results, patient information, and anatomic information. Reports and summary statistics can be extracted from the database and formatted into an HTML document.

Acknowledgements: This work was supported in part by HL64368-01 and HL60158-02 from the National Institutes of Health, by an NSF CAREER award, and by a Biomedical Engineering Research Grant from the Whitaker Foundation. 


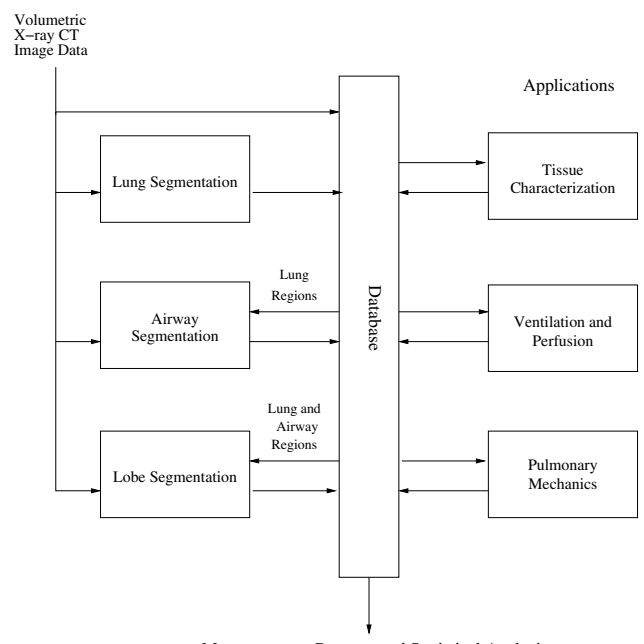

Measurements, Reports, and Statistical Analysis

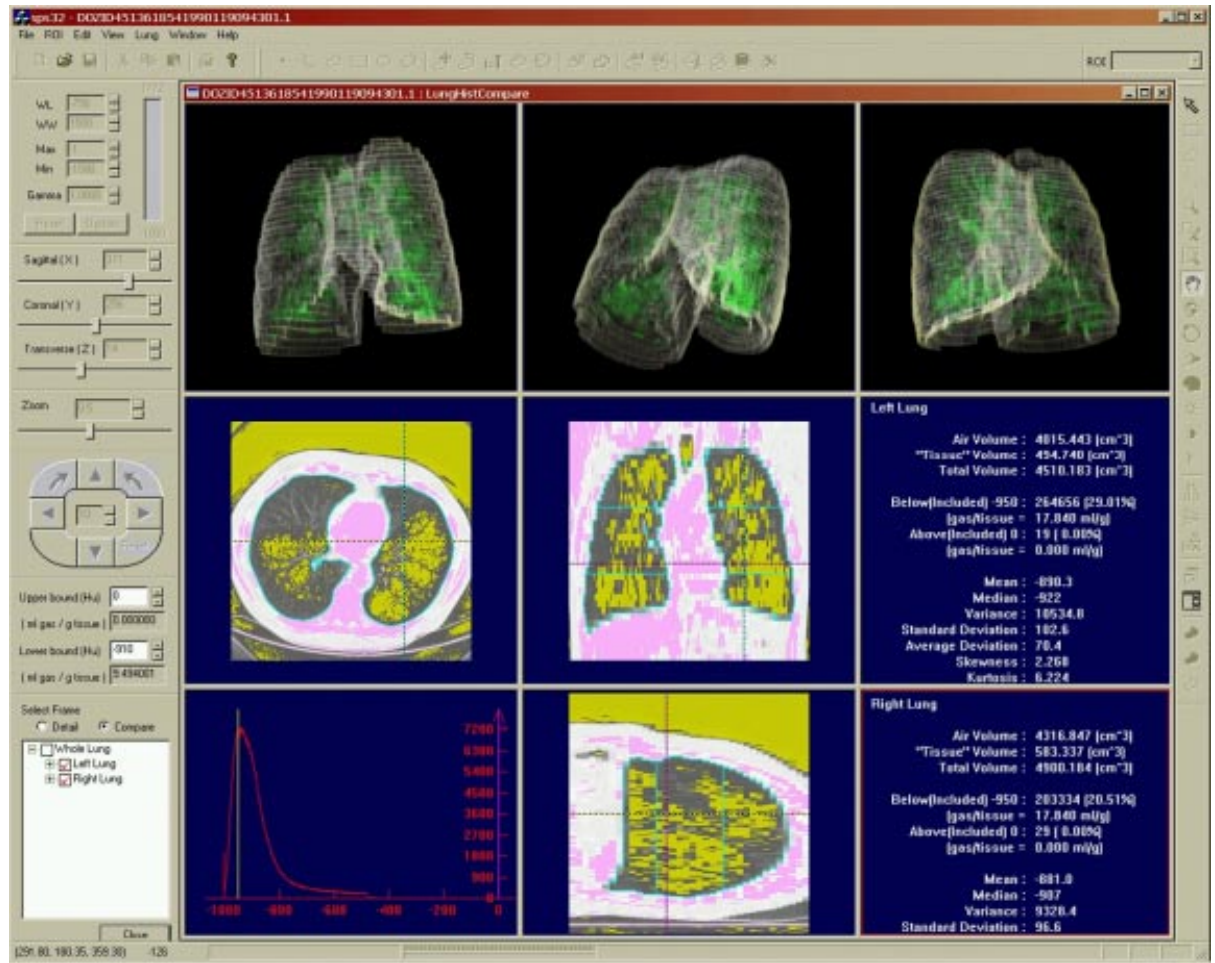

Fig. 1. Top: System architecture. Bottom: One view of analysis system showing volume-rendered views, cross-sections highlighting suspected pathology in green, pixel histograms, and textual summary statistics. 